\title{
TO OUR READERS
}

Starting with this issue the International Review of Social History will be directed by a new editorial committee, consisting half of members of the staff of the Internationaal Instituut voor Sociale Geschiedenis in Amsterdam, and half of scholars from Dutch universities. Shortly, this body is to be supported by an advisory board of an international character.

These changes in organization will be accompanied by a renewal of editorial policy, which will be expounded more extensively in the IRSH in the near future.

Marcel van der Linden 